\title{
Futuristic review on progress in force degradation studies and stability indicating assay method for some antiviral drugs
}

\author{
Jaya P. Ambhore ${ }^{1,}{ }^{*}$, Vaibhav S. Adhao ${ }^{1}$, Rameshwar S. Cheke ${ }^{1}$, Ritesh R. Popat ${ }^{2}$ and Shital J. Gandhi ${ }^{2}$ \\ ${ }^{1}$ Department of Pharmaceutical Chemistry, Dr. Rajendra Gode College of Pharmacy, Malkapur, Maharashtra, India. \\ 2 Department of Pharmaceutics, Dr. Rajendra Gode College of Pharmacy, Malkapur, Maharashtra, India.
}

GSC Biological and Pharmaceutical Sciences, 2021, 16(01), 133-149

Publication history: Received on 15 May 2021; revised on 13 July 2021; accepted on 15 July 2021

Article DOI: https://doi.org/10.30574/gscbps.2021.16.1.0172

\begin{abstract}
Force degradation studies of drug substance give perceptive knowledge about the intrinsic stability of the molecule as well as possible degradants which formed during the shelf life of drug and thus, aid within the successive development of its stable formulation. A number of analytical methods with hyphenated techniques are required for the identification, determination and characterization of degraded product and impurities produce during different conditions of stress studies; Chromatographic methodology play a vital role in the field of impurity and degradation profiling .This review summarizes the current regulatory requirements guidelines for the laboratory performance of forced degradation and its application for the development of stability indicating method. There are number of strategies have been implemented for the quantitative assessment of antiviral drugs. This study will provide detailed literature on stabilityindicating HPLC/ RP-HPLC approaches for the development and validation of various antiviral drugs.
\end{abstract}

Keywords: Intrinsic stability; Degradants; Forced degradation; Stability indicating methods

\section{Introduction}

The stability of pharmaceutical product requires more attention because the stability get directly affects the safety, purity and efficacy of drug products. Stability parameter of active pharmaceutical drugs and their formulations are determined during the early stage of drug development process [1]. The International Council for Harmonization (ICH) and Food and Drug Administration (FDA) provided the different guidelines and requirements for stability testing data [2]. According to different guidelines generally two types of stability testing studies, like, long-term stability studies and accelerated stability studies. In case of long-term studies, the require time for completion of study is about 12 months. Generally long-term stability studies useful for identification and separation of degraded products. In case of accelerated stability testing required around 6 months, Intermediate stability testing is also proceeding for 6 months at conditions milder than accelerated studies [3].

\section{Search area}

This review summarizes the current regulatory requirements for the practical performance of forced degradation and its application for the development of stability indicating method. There are numerous strategies have been implemented for the quantitative assessment of antiviral drugs. This study will provide detailed literature on stabilityindicating HPLC/ RP-HPLC approaches for the development and validation of various antiviral drugs as well as gives a basic idea to the researchers who are working in the area of product development and finish product testing.

\footnotetext{
* Corresponding author: Jaya P Ambhore

Department of Pharmaceutical Chemistry, Dr. Rajendra Gode College of Pharmacy, Malkapur, Maharashtra, India. 


\section{Discussion}

Authors selected many references published in the reputed journals on degradation studies, impurity profiling, and different stability indicating assay methods and took the important information from that is published in 1998-2021. The article are divided into various section such as regulatory requirements for the practical performance of forced degradation and development of stability indicating method detailed literature on stability- indicating HPLC/ RP-HPLC approaches for the development and validation of various antiviral drugs.

\section{Force degradation studies}

Force degradation studies are very useful to determine the reason of degradation routes and stability of pharmaceutical product under various stress producing conditions. In the force degradation studies the characterization of the degradants is usually performed with help of ICH guidelines. To performed stability studies and development of stability indicating method (SIM) various analytical equipment are used such as HPLC-UV and HPLC- (PDA) are two common equipment's, while characterization and structural information of degradant products LC-MS is most authentic method [4].

\section{Objective of forced degradation studies}

Forced degradation studies establish degradation pathways of drug molecule and their pharmaceutical products. For the structural elucidation of degradant force degradation studies play major role and also applicable for determination of intrinsic stability of a drug substance in pharmaceutical formulation. Forced degradation reveal the degradation mechanisms of the drug product and drug substance.These studies provided information about stability indicating nature of a developed method [5-7].

\section{Worldwide status of regulatory requirements on degradation studies}

For providing information about forced degradation many guidelines are mentioned like International Committee for harmonization (ICH), Food and drug administration (FDA), European Medicines Agency (EMA), United States Pharmacopeia (USP), Japanese Pharmacopoeia (JP), and Agencia National de Vigilancia Sanitaria (ANVISA). [8-9]

\subsection{ICH Guidelines}

In ICH guidelines ICH Q1A, Q1B, \& Q2B, Q3A, Q3B, M4Q (R1) discusses about Forced degradation studies.

\subsection{ICH Q1A - Testing of Stability for New drug molecules \& Products.}

ICH Q1A guideline gives information about stress testing of a drug product as the studies base on drug component and drug products. To carry out forced degradation evaluation on drug substances \& Products at several accelerated conditions were mentioned. Those conditions were effect of humidity (\% Relative humidity), oxidation, temperature, and photolysis \& range of $\mathrm{pH}$ solution / suspension) [10-11].

\subsection{ICH Q1B - Photostability Testing of New drug substances and drug products}

These guidelines use for check out the photostability behavior of drug substance in the development stage. The guideline provides information about the approaches to evaluate photostability of the drug and its formulation to be used in development of stability indicating methods $[11,12]$.

\subsection{ICH Q2B - Validation of analytical procedures: Methodology}

The ICH Q2 guideline gives the information about the protocols to be followed by validation of different analytical protocols. Sample usages for forced degradation studies mentioned in ICH Q2B, Part II, Section 1.2.2. Samples should be to stress under different accelerating condition like heat \& humidity used for determination of specificity. In additional, essential for the quantitative determination of the degradation produced [11, 13-15].

\subsection{ICH Q3 -Impurities in New drug substances}

These guidelines give the knowledge about the determination of impurity present in new drug molecules. In these Section gives guidelines about different aspect like identification of impurities, types of impurities \& specification impurities analytical protocols of impurities. Mostly it provided important about, validation of chromatographic method 
of new batch of drug molecule. According to guidelines in case of trace molecules, stability limit should be more than $90 \%$ and hence about $10 \%$ degradation is sufficient $[12,16]$.

\subsection{ICH Q3B - Impurities in New Products}

This guideline gives knowledge about analytical methods. It is important for an analytical method to validate the specific or non - specific degradation products under various stress conditions [17]

\subsection{ICH M4Q (R1) - The common technical document for the registration of pharmaceuticals for human use: Module 3: Quality}

This guideline gives knowledge about various types of studies performed, used procedures and various outcomes of the studies. In outcomes of stability checking covers in Section 3.2.S.7.3. In these guidelines results gives in form of graphic, tabular, narrative format with the validation data [18].

\section{FDA Guidelines (Food and drug administration)}

This guideline explains how to analyze the photo stability of newer drug molecules and products (Q1B). Forced degradation studies, according to the FDA, should be carried out in normal development conditions. It talks about how samples degrade when they're exposed to light. These guidelines also aid in the development of Stability indicating method (SIM) and the summarization of data from validation studies, which are confirmatory studies. According to section 211.166(a) (3) of these guidelines, a SIM should be highly precise and capable of quantifying the amount of active ingredient present, whether these types of degradation products occur, as well as other components present in dosage form, without interference under stress. $\mathrm{pH}$, temperature, and oxygen are three stress conditions that are needed for forced degradation studies $[13,19]$.

\section{EMA Guidelines (European Medicines Agency)}

These rules are essential for the chemistry of active substances. It includes information on the types of experiments that were conducted, the protocols that were used, and the results that were obtained as a result of the study. In section 2.1 .2 of this guideline, stability checking for API and dosage type is discussed. It also contains information on retest dates and substance expiration dates. It also determined the analytical methods for implementation and evaluation, as well as intrinsic stability and degradation pathways. It also conducts stability tests on sensitive compounds such as photosensitive and hygroscopic drugs $[8,20]$.

\section{USP Pharmacopoeia: validation of compendia procedures}

This guideline gives the knowledge about the If no degradation criterion or contaminants are available, the specificity should be calculated by comparing the data to the results obtained degradation product or contaminants using a different method under the same accelerated conditions [3,19].

\section{Japanese Pharmacopoeia}

This guideline notes that the proposed method must be precise in order to classify and detect the amount of analyte present in the sample. Samples may be subjected to stress conditions for comparative studies, and degradation products can be used for further studies if reference standards are not available [19].

\section{ANVISA (National Health Surveillance agency)}

This guideline explains how to meet the criteria for stability and forced degradation. These guidelines protect manufacturers from liability for risks posed by and uses of different drug products. It was created with the aim of promoting public health. It also encourages states, districts, and municipalities to promote the Brazilian unified health system's principles in order to enhance overall quality of life [19].

\section{Factors Affecting Degradation}

The following are the various factors that cause drug product degradation. They are as follows [20-23]: 


\subsection{Moisture}

Water soluble substances can dissolve if there is moisture present or when comes in contact with any moisture; the molecules undergo physical and chemical changes as a result of formulation prone to degradation and lose its properties.

\subsection{Temperature}

Temperature fluctuations may have a negative impact on the drug's stability. The rate of drug hydrolysis improves as the temperature rises.

\subsection{Excipients}

Some excipients have been found to have high water content. This moisture can cause an increase in the amount of water in the formulation, affecting the drug's stability. Chemical interactions between the excipients and the drug material can lead to decreased stability in some cases.

\section{4. pH}

The rate of drug degradation by hydrolysis is significantly affected by $\mathrm{pH}$. To minimize this effect, drug formulations are carried out with buffer solutions of the highest pH stability.

\subsection{Oxygen}

Oxidation of certain drugs is increased when oxygen is present. Purging nitrogen or carbon dioxide from the storage container stabilizes drugs that decompose more quickly in the presence of oxygen.

\subsection{Light}

Some drugs are photolabile, meaning they degrade when exposed to light. The susceptibility to photolytic decomposition can be determined by comparing the stability of the substance in the presence of light to the stability of the substance when stored in the dark. It is also important to note that photolabile compounds should be kept in amber glass containers and kept in the dark.

\section{Time to perform forced degradation}

When it comes to the production of new drug compounds and new drug products, it's vital to know when to conduct forced degradation studies. Stress testing should be done during the phase III of the regulatory submission period, according to FDA guidelines. To assess stress experiments should be conducted in various pH solutions, with oxygen and light present, and at elevated temperatures and humidity levels [6]. The stress tests are carried out in a single batch. The findings should be summarized and reported on annually. Stress research on drug substance should be started early in the preclinical process or phase I of clinical trials to provide enough time for identifying degradation products and structure elucidation, as well as optimizing the stress response. Early stress analysis also provides timely guidelines for making improvements in the manufacturing process and the correct range of stability-indicating analytical procedures $[19,24]$.

\section{Limits for Forced degradation studies}

The majority of regulatory guidelines listed degradation limits, such as 5\% -20\% agreed requirements for chromatographic assay validation. Stability should be greater than $90 \%$, with $10 \%$ being sufficient for deterioration. In general, spiked sample mixtures of known degradation drug content \& drug products are used to observe drug product stability, deciding the product that is controlled during the degradation process [25].

\section{Sources of degradation products}

Impurities may come from a variety of sources, including degradation. Drug molecules degrade due to chemical instability under various stress conditions such as heat, humidity, isolation, $\mathrm{pH}$, storage, and transportation processes. Forced degradation should be accomplished through a variety of methods, including oxidation, heat, photolysis, and hydrolysis $[6,10]$. 


\section{Plan for selection of forced degradation condition}

The intrinsic stability of a drug substance and its product should be calculated under normal conditions such as $\mathrm{pH}$ and high temperatures. Along with this, the drug molecules were subjected to additional stress in order to investigate its stability. The solution containing the sample was refluxed for a specific amount of time to investigate the forced degradation. If the degradation substance and product were to be monitored at that period, the process would be interrupted, and further detection, isolation, and monitoring of the degradation product would be carried out. If no signs of degradation are monitored, the reaction time to monitor any signs of degradation will increase as time passes. [3, 6, 19, 26-28] The following conditions were used in the forced degradation studies presented in Table 1 and Figure 1.

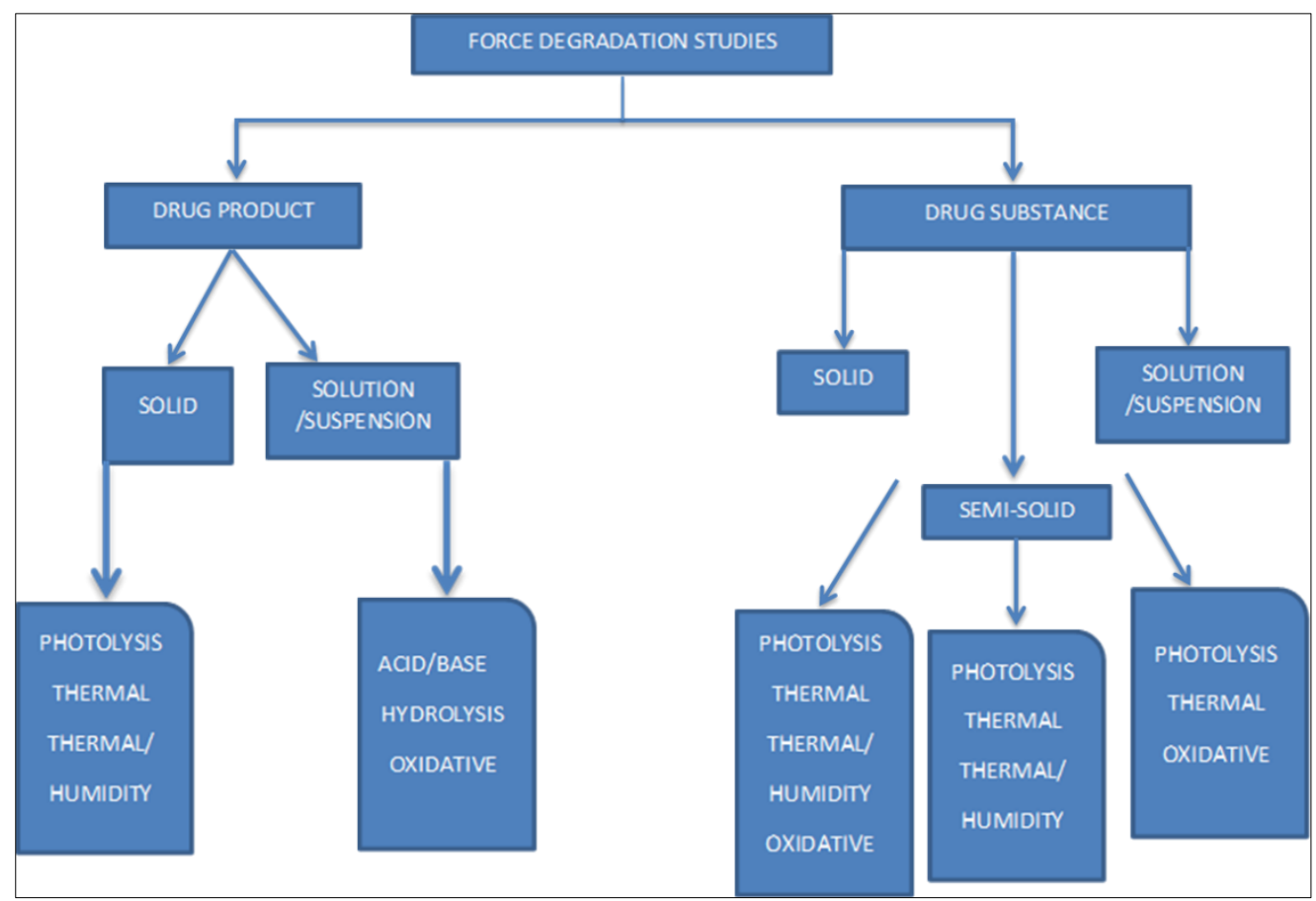

Figure 1 Representation of force degradation studies for drug product and drug substance 
Table 1 Condition used for forced degradation

\begin{tabular}{|c|c|c|c|c|}
\hline Degradation type & Experimental condition & Storage condition & Sampling time & References \\
\hline \multirow{6}{*}{ Hydrolysis } & Control API (no acid or base) & $40^{\circ} \mathrm{C}, 60^{\circ} \mathrm{C}$ & 1,3,5 Days & \multirow{18}{*}[3,6,19,26-28]{} \\
\hline & $0.1 \mathrm{~N} \mathrm{HCl}$ & $40^{\circ} \mathrm{C}, 60^{\circ} \mathrm{C}$ & 1,3,5 Days & \\
\hline & $0.1 \mathrm{~N} \mathrm{NaOH}$ & $40^{\circ} \mathrm{C}, 60^{\circ} \mathrm{C}$ & 1,3,5 Days & \\
\hline & Acid Control (no API) & $40{ }^{\circ} \mathrm{C}, 60^{\circ} \mathrm{C}$ & 1,3,5 Days & \\
\hline & Base Control (no API) & $40^{\circ} \mathrm{C}, 60^{\circ} \mathrm{C}$ & 1,3,5 Days & \\
\hline & $\mathrm{pH}: 2,4,6,8$ & $40^{\circ} \mathrm{C}, 60^{\circ} \mathrm{C}$ & 1,3,5 Days & \\
\hline \multirow{4}{*}{ Oxidative } & $3 \% \mathrm{H}_{2} \mathrm{O}_{2}$ & $25^{\circ} \mathrm{C}, 40^{\circ} \mathrm{C}$ & 1,3,5 Days & \\
\hline & Peroxide Control & $25^{\circ} \mathrm{C}, 40^{\circ} \mathrm{C}$ & 1,3,5 Days & \\
\hline & Azobisisobutyronitrile & $40^{\circ} \mathrm{C}, 60^{\circ} \mathrm{C}$ & 1,3,5 Days & \\
\hline & AIBN Control & $40^{\circ} \mathrm{C}, 60^{\circ} \mathrm{C}$ & 1,3,5 Days & \\
\hline \multirow{3}{*}{ Photolytic } & Light, $1 \times \mathrm{ICH}$ & NA & 1,3,5 Days & \\
\hline & Light, $3 \times \mathrm{ICH}$ & NA & 1,3,5 Days & \\
\hline & Light Control & NA & 1,3,5 Days & \\
\hline \multirow{5}{*}{ Thermal } & Heat Chamber & $60^{\circ} \mathrm{C}$ & 1,3,5 Days & \\
\hline & Heat Chamber & $60{ }^{\circ} \mathrm{C} / 75 \% \mathrm{RH}$ & 1,3,5 Days & \\
\hline & Heat Chamber & $80^{\circ} \mathrm{C}$ & 1,3,5 Days & \\
\hline & Heat Chamber & $80^{\circ} \mathrm{C} / 75 \% \mathrm{RH}$ & 1,3,5 Days & \\
\hline & Heat Control & Room temp & 1,3,5 Days & \\
\hline
\end{tabular}


Table 2 Summary of stability-indicating RP-HPLC methods of different antiviral drugs

\begin{tabular}{|c|c|c|c|c|c|c|c|c|}
\hline Sr.no & $\begin{array}{l}\text { Name of } \\
\text { Drug }\end{array}$ & Sample & Column & Mobile phase & Flow rate & Detection & Linearity & References \\
\hline \multirow[t]{2}{*}{1} & $\begin{array}{l}\text { Atazanavir } \\
\text { sulphate }\end{array}$ & API & $\begin{array}{l}\text { C18Phenomenex } \\
(250 \mathrm{~mm} \times 4.6 \mathrm{~mm}, 5 \\
\mu \mathrm{m})\end{array}$ & Methanol: water $(900: 100)$ & $0.5 \mathrm{ml} / \mathrm{min}$ & $249 \mathrm{~nm}$ & $\begin{array}{l}10-90 \\
\mu \mathrm{g} / \mathrm{ml}\end{array}$ & {$[35]$} \\
\hline & $\begin{array}{l}\text { Atazanavir } \\
\text { sulphate }\end{array}$ & Capsule & $\begin{array}{l}\text { Agilent TC C18 (2) } \\
250 \times 4.6 \mathrm{~mm}, 5 \mu\end{array}$ & $\begin{array}{l}0.02 \mathrm{M} \text { ammonium dihydrogen } \\
\text { phosphate buffer: acetonitrile: } \\
\text { methanol }(30: 25: 45 \mathrm{v} / \mathrm{v})\end{array}$ & $1 \mathrm{ml} / \mathrm{min}$ & $288 \mathrm{~nm}$ & $5-50 \mu \mathrm{g} / \mathrm{ml}$ & [36] \\
\hline \multirow[t]{2}{*}{2} & Abacavir & API+ Tablet & $\begin{array}{l}\text { C18- column, } \\
\text { Phenomenex Luna, } \\
(250 \times 4.6 \mathrm{~mm}, 5 \mu \mathrm{m})\end{array}$ & Water: acetonitrile $(80: 20)$ & $1 \mathrm{ml} / \mathrm{min}$ & $285 \mathrm{~nm}$ & $\begin{array}{l}600-1600 \\
\mathrm{ng} / \mathrm{ml}\end{array}$ & {$[37]$} \\
\hline & $\begin{array}{l}\text { Abacavir } \\
\text { sulfate }\end{array}$ & Tablet & Grace C18 column & $\begin{array}{l}\text { Methanol and } 10 \mathrm{ml} \text { of potassium } \\
\text { dihydrogen orthophosphate }(38: 62 \\
\text { v/v). }\end{array}$ & $0.9 \mathrm{ml} / \mathrm{min}$ & $255 \mathrm{~nm}$ & $\begin{array}{l}10-50 \\
\mathrm{mg} / \mathrm{mL}\end{array}$ & {$[38]$} \\
\hline \multirow[t]{2}{*}{3} & Acyclovir & API & $\begin{array}{l}\text { Nova Pack C18 }(250 \\
x \text { 4.6) } \mathrm{mm}, 5 \mu\end{array}$ & $\begin{array}{l}\text { Buffer, methanol and acetonitrile } \\
(50: 20: 30, v / v / v)\end{array}$ & $1.0 \mathrm{~mL} / \mathrm{min}$ & $264 \mathrm{~nm}$ & $\begin{array}{l}80-120 \\
\mu \mathrm{g} / \mathrm{ml}\end{array}$ & [39] \\
\hline & Acyclovir & $\begin{array}{l}\text { API+ } \\
\text { Ointment }\end{array}$ & $\begin{array}{ll}\text { Agilent } & \text { column } \\
(150 \mathrm{mmx} & 4.6 \mathrm{~mm}, \\
5 \mu \mathrm{m}) & \end{array}$ & $\begin{array}{l}\text { Acetonitrile, Methanol and } \\
\text { phosphate buffer }(16: 20: 64 \mathrm{v} / \mathrm{v})\end{array}$ & $1.0 \mathrm{~mL} / \mathrm{min}$ & $290 \mathrm{~nm}$ & $\begin{array}{l}20-100 \\
\mu \mathrm{g} / \mathrm{ml}\end{array}$ & {$[40]$} \\
\hline 4 & $\begin{array}{l}\text { Adefovir } \\
\text { Dipivoxil }\end{array}$ & API+Tablet & $\begin{array}{l}\text { Nucleodur } \quad \text { C18 } \\
\text { column }(15 \mathrm{~cm} \times 4.6 \\
\text { mm i.d.) }\end{array}$ & $\begin{array}{l}\text { Acetonitrile-citrate buffer (10 mM } \\
\text { at pH 5.2) } 36: 64(\% \mathrm{v} / \mathrm{v})\end{array}$ & $1.5 \mathrm{~mL} / \mathrm{min}$ & $260 \mathrm{~nm}$ & $\begin{array}{l}0.5-16 \\
\mu \mathrm{g} / \mathrm{mL}\end{array}$ & {$[41]$} \\
\hline 5 & Boceprevir & API & $\begin{array}{l}\text { HiQsil C-18 Column } \\
(150 \mathrm{~mm} \times 4.6 \mathrm{~mm} \\
\text { i.d., particle size } 5 \\
\mu \mathrm{m})\end{array}$ & $\begin{array}{l}\text { Methanol :10mM dihydrogen } \\
\text { phosphate: } \\
(90: 10: 0.5)(\% \mathrm{v} / \mathrm{v} / \mathrm{v})\end{array}$ & $1.0 \mathrm{~mL} / \mathrm{min}$ & $215 \mathrm{~nm}$ & $\begin{array}{l}50-250 \\
\mu \mathrm{g} / \mathrm{mL}\end{array}$ & [42] \\
\hline 6 & $\begin{array}{l}\text { Baloxavir } \\
\text { marboxil }\end{array}$ & Tablet & $\begin{array}{l}\text { HSS C18 (100 x } 2.1 \\
\mathrm{~mm}, 1.8 \mathrm{~mm})\end{array}$ & $\begin{array}{l}\text { Buffer } 0.1 \% \text { orthophosphoric acid } \\
\text { and acetonitrile }(50: 50)\end{array}$ & $0.3 \mathrm{ml} / \mathrm{min}$. & $240 \mathrm{~nm}$ & $\begin{array}{l}0.69-2.10 \\
\mathrm{mg} / \mathrm{ml}\end{array}$ & [43] \\
\hline
\end{tabular}




\begin{tabular}{|c|c|c|c|c|c|c|c|c|}
\hline 7 & Cobicistat & API+ Tablet & $\begin{array}{l}\text { Hypersil } \text { BDS } \quad \text { C-18 } \\
(150 \mathrm{~mm} \\
5 \mu)\end{array}$ & Water: Acetonitrile (90:10) & $1.0 \mathrm{ml} / \mathrm{min}$ & $240 \mathrm{~nm}$ & $\begin{array}{l}7.5 \\
45 \mu \mathrm{g} / \mathrm{mL}\end{array}$ & {$[44]$} \\
\hline \multirow[t]{2}{*}{8} & $\begin{array}{l}\text { Darunavir } \\
\text { ethanolate }\end{array}$ & API+ Tablet & $\begin{array}{l}\text { X-Bridge C18 }(150 \times \\
4.6 \mathrm{~mm} \times 3.5 \mu \mathrm{m})\end{array}$ & $\begin{array}{l}0.01 \mathrm{M} \text { ammonium formate }(\mathrm{pH} .3 .0) \\
\text { buffer }+ \text { acetonitrile in the ratio of } \\
55: 45(\mathrm{v} / \mathrm{v})\end{array}$ & $1.0 \mathrm{ml} / \mathrm{min}$ & $265 \mathrm{~nm}$ & $\begin{array}{l}0.16 \text { to } 0.24 \\
\mathrm{mg} / \mathrm{mL}\end{array}$ & {$[45]$} \\
\hline & $\begin{array}{l}\text { Darunavir } \\
\text { ethanolate }\end{array}$ & $\begin{array}{l}\text { Raw material } \\
+ \text { Tablet }\end{array}$ & $\begin{array}{l}\text { RP C18 column } \\
\text { (Symmetry, } \\
5 \mu \mathrm{m}, \mathrm{mm} \text {, Waters) }\end{array}$ & acetonitrile water $(50: 50, \mathrm{v} / \mathrm{v})$ & $1.0 \mathrm{ml} / \mathrm{min}$ & $267 \mathrm{~nm}$ & $\begin{array}{l}6.0 \quad \text { to } \\
21.0 \mu \mathrm{g} / \mathrm{mL}\end{array}$ & {$[46]$} \\
\hline \multirow[t]{2}{*}{9} & $\begin{array}{l}\text { Dolutegravir } \\
\text { sodium }\end{array}$ & API+ Tablet & $\begin{array}{l}\text { ODS } \mathrm{C}_{18} \text { column }(150 \\
\times \quad 4.6 \mathrm{~mm}, \quad 5 \mu \mathrm{m} \\
\text { particle size })\end{array}$ & $\begin{array}{l}\text { acetonitrile: water }(\mathrm{pH} 7.5) \text { in the } \\
\text { ratio of } 80: 20 \mathrm{v} / \mathrm{v}\end{array}$ & $1 \mathrm{ml} / \mathrm{min}$ & $260 \mathrm{~nm}$ & $\begin{array}{l}5-35 \\
\mu \mathrm{g} / \mathrm{mL}\end{array}$ & {$[47]$} \\
\hline & $\begin{array}{l}\text { Dolutegravir } \\
\text { sodium }\end{array}$ & API+ Tablet & $\begin{array}{l}\text { C8 column }(150 \times 4.6 \\
\mathrm{mm}), 5 \mu \mathrm{m}\end{array}$ & $\begin{array}{l}0.1 \% \text { trifluoroacetic acid in water as } \\
\text { MP: A, methanol as MP: B. }\end{array}$ & $0.9 \mathrm{ml} / \mathrm{min}$ & $240 \mathrm{~nm}$ & $0.1-0.5 / \mathrm{mL}$ & {$[48]$} \\
\hline 10 & Didanosin & Tablet & $\begin{array}{l}\text { Zebra Eclipse XDB-C- } \\
18(4.6 \times 250 \times 5 \mu \mathrm{m})\end{array}$ & methanol: water $(30: 70)$ & $1.0 \mathrm{ml} / \mathrm{min}$ & $246 \mathrm{~nm}$ & $\begin{array}{l}2-12 \mu \mathrm{g} / \mathrm{ml} \\
\mathrm{w}\end{array}$ & [49] \\
\hline \multirow[t]{3}{*}{11} & Efavirenz & Tablet & $\begin{array}{l}\text { Agilent eclipse XDB } \\
\text { C18 column }\end{array}$ & Acetonitrile: Water $(60: 40 \mathrm{v} / \mathrm{v})$ & $1.2 \mathrm{ml} / \mathrm{min}$ & $240 \mathrm{~nm}$ & $\begin{array}{l}2.5- \\
200.0 \mu \mathrm{g} / \mathrm{mL}\end{array}$ & {$[50]$} \\
\hline & Efavirenz & API & $\begin{array}{l}\text { RP Spherisorb C-8 } \\
\text { (Waters) } \quad \text { column } \\
(250 \times 4.6 \mathrm{~mm}, 10 \\
\mu \mathrm{m}),\end{array}$ & $\begin{array}{l}\text { Acetonitrile: potassium dihydrogen } \\
\text { phosphate }(\mathrm{pH} \quad 2.9,25 \mathrm{mM}) \\
(60: 40 \% \mathrm{v} / \mathrm{v})\end{array}$ & $\begin{array}{l}1.0 \\
\mathrm{ml} / \mathrm{min}\end{array}$ & $247 \mathrm{~nm}$ & $\begin{array}{l}0.02-20.00 \\
\mu \mathrm{g} \mathrm{mL}-1\end{array}$ & [51] \\
\hline & Efavirenz & API + Tablet & 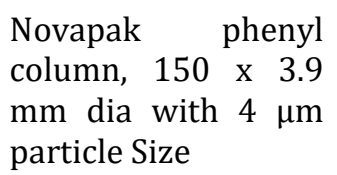 & $\begin{array}{l}\text { Buffer solution pH } 6.0 \text { and } \\
\text { acetonitrile in proportion }(55: 45 \\
\mathrm{v} / \mathrm{v})\end{array}$ & $\begin{array}{l}1.0 \\
\mathrm{ml} / \mathrm{min}\end{array}$ & $247 \mathrm{~nm}$ & $\begin{array}{l}0.05-0.15 \\
\mathrm{mg} / \mathrm{mL}\end{array}$ & {$[52]$} \\
\hline 12 & Emtricitabine & API & $\begin{array}{l}\text { Intersil } \quad \text { ODS-3V } \\
\text { column }\end{array}$ & $\begin{array}{l}10 \mathrm{mM} \text { sodium phosphate buffer } \\
\text { and methanol }(85: 15)\end{array}$ & $\begin{array}{l}1.0 \\
\mathrm{ml} / \mathrm{min}\end{array}$ & $280 \mathrm{~nm}$ & $\begin{array}{l}0.002 \text { to } 0.5 \\
\mathrm{mg} / \mathrm{mL} .\end{array}$ & [53] \\
\hline 13 & Etravirine & API & $\begin{array}{l}\text { Zorbax Eclipse Plus } \\
\text { C18 }(250 \times 4.6 \mathrm{~mm} ; 5 \\
\mu \mathrm{m} \text { particle size })\end{array}$ & $\begin{array}{l}\text { acetonitrile and } 10 \mathrm{mM} \text { ammonium } \\
\text { acetate buffer (pH } 4.5) \text { in the ratio } \\
\text { of } 90: 10 \mathrm{v} / \mathrm{v} \text {. }\end{array}$ & $\begin{array}{l}1.0 \\
\mathrm{~mL} / \mathrm{min}\end{array}$ & $271 \mathrm{~nm}$ & $\begin{array}{l}15-45 \\
\mu \mathrm{g} / \mathrm{ml}\end{array}$ & [54] \\
\hline
\end{tabular}




\begin{tabular}{|c|c|c|c|c|c|c|c|c|}
\hline & Etravirine & API & $\begin{array}{l}\text { hypersil ods C18, } \\
(150 * 4.6 \mathrm{~mm}, 5 \mu)\end{array}$ & Acetonitrile & $\begin{array}{l}1.0 \\
\mathrm{ml} / \mathrm{min}\end{array}$ & $271 \mathrm{~nm}$ & $\begin{array}{l}10-60 \\
\mu \mathrm{g} / \mathrm{ml}\end{array}$ & {$[55]$} \\
\hline \multirow[t]{3}{*}{14} & Famciclovir & API & $\begin{array}{l}\text { RP-C18 column } 250 \\
\mathrm{~mm} \times 4.6 \mathrm{~mm} \text { i. d., } 5- \\
\mu \mathrm{m}\end{array}$ & $\begin{array}{l}\text { Methanol: KH2PO4 buffer (pH 3.0, } \\
\text { with ortho phosphoric acid) (35:65, } \\
\mathrm{v} / \mathrm{v} \text { ) }\end{array}$ & $1.0 \mathrm{ml} / \mathrm{min}$ & $242 \mathrm{~nm}$ & $\begin{array}{l}10-50.00 \mu \mathrm{g} \\
\mathrm{mL}-1\end{array}$ & [56] \\
\hline & Famciclovir & API + Tablet & $\begin{array}{lr}\text { C18- } & \text { column } \\
\text { Hypersil } & \text { BDS } \\
(250 \times 4.6 \mathrm{~mm}, 5 \mu)\end{array}$ & 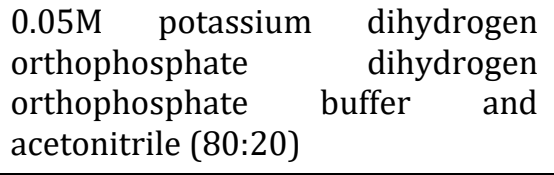 & $1.0 \mathrm{ml} / \mathrm{min}$ & $220 \mathrm{~nm}$ & $\begin{array}{l}0.025- \\
0.150 \mathrm{mg} / \mathrm{ml}\end{array}$ & {$[57]$} \\
\hline & Famciclovir & API & $\begin{array}{l}\text { C18- column, } \\
\text { Symmetry } \\
\text { c18(150×4.6 mm, } 5 \\
\mu)\end{array}$ & $\begin{array}{l}\text { Mixture of phosphate buffer }(0.02 \mathrm{M} \\
\text { pH: } 5.0 \pm 0.050 \text { with dilute } \\
\text { orthophosphoric acid acetonitrile } \\
(80: 20)\end{array}$ & $1.0 \mathrm{ml} / \mathrm{min}$ & $220 \mathrm{~nm}$ & $20-30 \mu \mathrm{g} / \mathrm{ml}$ & {$[58]$} \\
\hline 15 & Foscarnet & $\begin{array}{l}\text { API+ } \\
\text { Injection }\end{array}$ & $\begin{array}{l}\text { C18- column, ODS } \\
(150 \times 4.6 \mathrm{~mm}, 5 \mu)\end{array}$ & Buffer and acetonitrile (40:60) & $1.1 \mathrm{ml} / \mathrm{min}$ & $225 \mathrm{~nm}$ & $\begin{array}{l}0.25-1.5 \\
\mu \mathrm{g} / \mathrm{ml}\end{array}$ & [59] \\
\hline 16 & Ganciclovir & API & $\begin{array}{l}\text { BDS C18, } 150 \mathrm{~mm} \mathrm{X} \\
\text { four. } 6 \mathrm{~mm}, 5 \mu \mathrm{m}\end{array}$ & $\begin{array}{l}\text { Phosphate buffer and acetonitrile } \\
70: 30(\mathrm{v} / \mathrm{v})\end{array}$ & $1.0 \mathrm{ml} / \mathrm{min}$ & $245 \mathrm{~nm}$ & $\begin{array}{l}20-30 \mu g \\
m L\end{array}$ & [60] \\
\hline 17 & Imiquimod & API & $\begin{array}{l}\text { C18 column (Inertsil- } \\
\text { ODS3 } 4.6 \times 250 \mathrm{~mm} \text {, } \\
5 \mu \mathrm{m})\end{array}$ & $\begin{array}{l}\text { Phase-A disodium hydrogen } \\
\text { phosphate buffer with ortho- } \\
\text { phosphoric acid. mobile phase-B } \\
\text { methanol and acetonitrile }\end{array}$ & $1.2 \mathrm{ml} / \mathrm{min}$ & $226 \mathrm{~nm}$ & $0 \%$ to $150 \%$ & [61] \\
\hline 18 & Lamivudine & API + Tablet & $\begin{array}{l}\text { IntersilC-18 }(250 \quad X \\
4.6 \mathrm{~mm}, 5 \mu \mathrm{m}) \text { column }\end{array}$ & Acetonitrile, water (50:50\% v/v) & $1.0 \mathrm{ml} / \mathrm{min}$ & $270 \mathrm{~nm}$ & $\begin{array}{l}40-120 \\
\mu \mathrm{g} / \mathrm{ml}\end{array}$ & {$[62]$} \\
\hline 19 & Oseltamivir & API & $\begin{array}{l}\text { Kromasil C (18), } 5 \\
\text { microm, } 250 \mathrm{~mm} \mathrm{x} \\
4.6 \mathrm{~mm}\end{array}$ & Acetonitrile and triethylamine) & $1.0 \mathrm{ml} / \mathrm{min}$ & $215 \mathrm{~nm}$ & $\begin{array}{l}70-130 \\
\text { microg/ml }\end{array}$ & {$[63]$} \\
\hline 20 & Ribavirin & $\begin{array}{l}\text { Capsule+ } \\
\text { Plasma }\end{array}$ & $\begin{array}{l}\text { CPS Hypersil cyano } \\
\text { column }(4.6 \times 250 \\
\mathrm{mm} \times 5 \mu \mathrm{m})\end{array}$ & $\begin{array}{l}50 \mathrm{mM} \text { phosphate buffer of } \mathrm{pH} 4 \\
\text { (adjusted by using phosphoric } \\
\text { acid). }\end{array}$ & $0.8 \mathrm{ml} / \mathrm{min}$ & $240 \mathrm{~nm}$ & $\begin{array}{l}5-200 \\
\mu \mathrm{g} / \mathrm{mL}\end{array}$ & [64] \\
\hline
\end{tabular}




\begin{tabular}{|c|c|c|c|c|c|c|c|c|}
\hline & Ribavirin & API & $\begin{array}{l}\text { Promosil } \\
\text { stainless } \\
\text { column }(250 \times 4.6 \\
\text { sm, } 5 \mu \mathrm{m} \text { particle } \\
\text { size })\end{array}$ & $\begin{array}{l}0.02 \mathrm{M} \text { potassium dihydrogen } \\
\text { phosphate of } \mathrm{pH} 4.7\end{array}$ & $1.1 \mathrm{ml} / \mathrm{min}$ & $207 \mathrm{~nm}$ & $\begin{array}{l}2.0-40 \\
\mu \mathrm{g} / \mathrm{ml}\end{array}$ & [65] \\
\hline \multirow[t]{2}{*}{21} & Simeprevir & API & $\begin{array}{l}\text { Discovery }{ }^{\circledR} \text { HS } \quad \text { C18 } \\
\text { column }\end{array}$ & Acetonitrile. & $1.0 \mathrm{ml} / \mathrm{min}$ & $288 \mathrm{~nm}$ & $\begin{array}{l}1.5-45 \\
\mu \mathrm{g} / \mathrm{mL} .\end{array}$ & [66] \\
\hline & Simeprevir & API & $\begin{array}{l}\text { C18 column } \\
(250 \times 4.6,5 \mathrm{~mm}\end{array}$ & $\begin{array}{l}\text { orthophosphoric acid and } \\
\text { acetonitrile }(55: 45 \% \mathrm{v} / \mathrm{v})\end{array}$ & $1.0 \mathrm{ml} / \mathrm{min}$ & $300 \mathrm{~nm}$ & $\begin{array}{l}25-150 \\
\mu \mathrm{g} / \mathrm{ml}\end{array}$ & [67] \\
\hline 22 & Tenofovir & $\begin{array}{l}\text { Solid lipid } \\
\text { nanoparticles }\end{array}$ & $\begin{array}{l}\text { C18- column Agilent } \\
(250 \times 4.6 \mathrm{~mm} \times 5 \mu \mathrm{m})\end{array}$ & $\begin{array}{l}20 \mathrm{mM} \text { potassium dihydrogen } \\
\text { orthophosphate and acetonitrile, } \\
(50: 50)\end{array}$ & $1.0 \mathrm{ml} / \mathrm{min}$ & $260 \mathrm{~nm}$ & $\begin{array}{l}10-60 \\
\mu \mathrm{g} / \mathrm{ml}\end{array}$ & [68] \\
\hline \multirow[t]{4}{*}{23} & Valganciclovir & $\begin{array}{l}\text { API and } \\
\text { Tablet }\end{array}$ & $\begin{array}{l}\text { C18- column, HSS } \\
(100 \times 2.1 \mathrm{~mm}, 1.8 \mathrm{~m})\end{array}$ & $\begin{array}{l}0.01 \mathrm{~N} \text { potassium dihydrogen } \\
\text { orthophosphate and acetonitrile } \\
(55: 45)\end{array}$ & $0.3 \mathrm{ml} / \mathrm{min}$ & $254 \mathrm{~nm}$ & $\begin{array}{l}25-150 \\
\mu \mathrm{g} / \mathrm{m}\end{array}$ & [69] \\
\hline & Valganciclovir & API & $\begin{array}{l}\text { C18- rolumn, } \\
\text { Hypersil gold } \\
(250 \times 4.6 \mathrm{~mm}, 5 \mu \mathrm{m}\end{array}$ & $\begin{array}{l}\text { Acetonitrile and potassium } \\
\text { dihydrogen orthophosphate buffer } \\
\text { (pH 5.0) in the ratio of 5:95 }\end{array}$ & $0.6 \mathrm{ml} / \mathrm{min}$ & $252 \mathrm{~nm}$ & $\begin{array}{l}2-500 \\
\mu \mathrm{g} / \mathrm{ml}\end{array}$ & [70] \\
\hline & Valganciclovir & API & $\begin{array}{l}\text { C18- column, Hibar } \\
(250 \times 4.6 \mathrm{~mm}, 5 \mu)\end{array}$ & $\begin{array}{l}10 \text { volumes of methanol and } 90 \\
\text { volumes of ammonium acetate }\end{array}$ & $1 \mathrm{ml} / \mathrm{min}$ & $254 \mathrm{~nm}$ & $\begin{array}{l}18-72 \\
\mu \mathrm{g} / \mathrm{ml}\end{array}$ & [71] \\
\hline & Valganciclovir & API & $\begin{array}{l}\text { C18- column, } \mu- \\
\text { Bondapack }(250 \times 4.6 \\
\mathrm{mm}, 5 \mu)\end{array}$ & $\begin{array}{l}0.01 \mathrm{~N} \text { sodium dihydrogen } \\
\text { phosphate buffer (pH 5.0) and } \\
\text { acetonitrile in the ratio of 600:400 }\end{array}$ & $1.2 \mathrm{ml} / \mathrm{min}$ & $254 \mathrm{~nm}$ & $5-30 \mu \mathrm{g} / \mathrm{ml}$ & [72] \\
\hline 24 & Zanamivir & API & $\begin{array}{l}\text { C18- column, Agilent } \\
\text { TC } 250 \times 4.6 \mathrm{~mm}, 5 \mu \text { ) }\end{array}$ & $\begin{array}{l}\text { Methanol:0.02M phosphate buffer, } \\
\text { pH -3.5 (50:50) }\end{array}$ & $1.0 \mathrm{ml} / \mathrm{min}$ & $230 \mathrm{~nm}$ & $2-12 \mu \mathrm{g} / \mathrm{ml}$ & [73] \\
\hline 25 & Zidovudine & API+ Tablet & $\begin{array}{l}\text { Phenomenex, }(250 \times \\
4.6 \mathrm{~mm}) \text { Luna } 5 \mathrm{~m} \\
\text { C18 Column. }\end{array}$ & Methanol +Water $(89: 11 \mathrm{v} / \mathrm{v})$ & $1.0 \mathrm{ml} / \mathrm{min}$ & $266 \mathrm{~nm}$ & $\begin{array}{l}25-350 \\
\mu \mathrm{g} / \mathrm{ml}\end{array}$ & [74] \\
\hline
\end{tabular}




\section{Forced degradation condition}

\subsection{Hydrolysis}

The drug reacts with water under various $\mathrm{pH}$ conditions during hydrolysis (both acidic and alkaline). The drug compounds are generally prepared at $50-60^{\circ} \mathrm{C}$ with $0.1 \mathrm{~N}$ hydrochloric acid, sulphuric acid, or $0.1 \mathrm{~N}$ sodium hydroxide. The strength of the acid or alkali used in the experiment determines the molecule's stability. The intensity of acid or alkali solutions should be kept between 0.1 and $1 \mathrm{~N}$. Notably, the analysis does not last longer than 7 days. To prevent decomposition, samples should be neutralized with buffer or acid/base after being exposed to stress conditions.

\subsection{Oxidation}

Auto-oxidizers are existing in the majority of drug substances. For the oxidation phase, they need free radical initiators. Free radical initiators consist of hydrogen peroxide, trace impurities, and metal ions. The transfer of electrons is involved in this form of degradation $.0 .1-3 \%$ of hydrogen peroxide is a frequent initiator for oxidation forced degradation studies. These research need to be carried out at $40^{\circ} \mathrm{C}$ for $1-7$ days. If greater than $20 \%$ degradants are produced, then it needs to be considered as abnormal.

\subsection{Thermal condition}

Some drugs are viewed to be thermolabile in nature. By increasing the temperature, the rate of response additionally tends to increase which in turn leads to the production of degradation products. These researches have to be performed at $40-80^{\circ} \mathrm{C}$. The periods of thermal stress studies generally lasts for $1-2$ months and are carried out at $70^{\circ} \mathrm{C}$ and at high humidity. The drug molecules which are solid in nature are subjected to each dry and wet heat conditions, whilst liquids are exposed to dry heat for the shorter duration of time. Due to the elevated temperature, the drug molecule undergoes degradation and given by Arrhenius equation:

$$
\mathrm{k}=\frac{\mathrm{Ae}-\mathrm{Ea}}{\mathrm{RT}}
$$

Where,

K: Specific reaction rate, A: Frequency factor, Ea: Energy of activation, R: Gas constant (1.987 cal/ deg/mole), and T: Absolute temperature in Kelvin.

\subsection{Photolytic conditions}

In photolytic degradation studies, the drug substances are exposed to UV or fluorescent conditions. In this study, the drug components or drug products (solid/ liquid) are exposed to the light supply in accordance to the ICH Q1B protocols. The generally used radiation vary for degradation research is about $300-800 \mathrm{~nm}$. In photolytic condition, the degradation happens due to oxidation via free radical mechanism or non-oxidation process. Non-oxidative degradation process involves with isomerization, dimerization, etc., amongst others. On the other hand, oxidative photolytic response includes mechanism involving singlet/triplet oxygen states. Singlet oxygen reacts with unsaturated compounds to produce photooxidative decomposition products, while triplet oxygen follows free radical mechanism, to produce peroxide. Notably, it is proven that light also catalyzes oxidation reactions. In non-oxidative process, numerous types of reactions are discovered such as the hemolytic breakage of $\mathrm{C}$-X hetero bonds, deamination, and cleavage of C-S bonds are observed.

\subsection{Stability Indicating Method}

A stability indicating method (SIM) is an analytical procedure used to quantitate the decrease in the amount of the active pharmaceutical ingredient (API) in drug product due to degradation. According to an FDA guidance document, a stability-indicating method is a validated quantitative analytical procedure that can be used to detect how the stability of the drug substances and drug products changes with time. A stability-indicating method accurately measures the changes inactive ingredients concentration without interference from other degradation products, impurities and excipients [29]. Stress testing is carried out to demonstrate specificity of the developed method to measure the changes in concentration of drug substance when little information is available about potential degradation product. The development of a suitable stability indicating method provides a background for the pre-formulation studies, stability studies and the development of proper storage requirements. The RP-HPLC is a most widely used analytical tool for separation and quantifying the impurities and it is most frequently coupled with a UV detector [30-31]. 


\section{Development of stability indicating method}

Though the necessities with respect to stability indicating technique have been spelt out in regulatory documents, records on the fundamental steps to be observed for the improvement and validation of stability-indicating methods is neither provided in the regulatory guidelines nor in the pharmacopoeias [30, 32-33].

\section{Step 1: Critical study of the drug structure to assess the likely decomposition route}

These have to be the first element every time one takes up the project on establishment of a SIM. Much information can clearly be gained from the structure, with the aid of study of the functional groups and other key components.

\section{Step 2: Collection of information on physicochemical properties}

Before method development is taken up, it is commonly essential to recognize more than a few physicochemical parameters like $\mathrm{pKa}, \log \mathrm{P}$, solubility, absorptivity and wavelength most of the drug .

\section{Step 3: Stress (forced decomposition) studies}

As described above in forced degradation section, these researches have to be carried out in accordance with ICH Q1A guideline. Stress conditions are (i) $10^{\circ} \mathrm{C}$ increments above the accelerated temperatures (e.g. $50^{\circ} \mathrm{C}, 60^{\circ} \mathrm{C}$, etc.), (ii) humidity where appropriate (e.g. 75\% or greater), (iii) hydrolysis throughout a wide range of pH values, (iv) oxidation and (v) photolysis.

\section{Step 4: Preliminary separation studies on stressed samples}

The simplest of separation way is to begin with a reversed-phase octadecyl column and perform HPLC separation using UV/PDA detector system. Another way is to go for LC-MS separation. Using these chromatographic techniques, one need to comply with the changes in all the stress samples at a variety of time periods. The effects need to be critically compared with the blank solutions injected in a comparable manner. It has to be found whether or not the fall in drug peak is quantitatively followed through a corresponding rise in the degradation product peaks.

\section{Step 5: Final method development and optimization}

Ensuing to fundamental chromatographic examinations, the RT and relative retention times (RRT) of substance shaped should be arranged for each response condition. Exceptional consideration is then paid to these viewpoints whose RT or RRT is close. PDA spectra or LC-MS profile of such viewpoints are gotten and altogether assessed to check whether the product is same or different. To isolate or separate close or co-eluting pecks, the technique is streamlined, by changing the $\mathrm{pH}$, ratio of solvent system, flow rate, mode, temperature, and column.

\section{Step 6: Identification and characterization of degradation products, and preparation of standards}

To perceive the resolved products, a conventional way is to isolate them and determine the structure via spectral (MS, NMR, IR, etc.) and elemental analysis. However, this strategy is tedious and time consuming when more than one degradation products are formed. Against it, the modern method is to use hyphenated LC methods coupled with mass spectrometry. This approach integrates in a single instrument approach; this method integrates in a single instrument approach, analytical HPLC, UV detection, full scan mass spectrometry (LC-MS) and tandem mass spectrometry (LC-MSMS) and presents a fair concept on identification of resolving components. These days an in addition integrated method is becoming popular whereby LC-MS or LC-MS-MS is employed to obtain molecular weight and fragmentation information, and in addition detailed structural data is got via LC-NMR analysis.

\section{Step 7: Validation}

Validation of analytical methods, in general, has been extensively included in the ICH guidelines Q2A and Q2B, in the FDA guidance and through USP [10-13]. The main focus of validation at this stage is on institution of specificity/selectivity, followed through different parameters like accuracy, precision, linearity, range, robustness, etc. The limits of detection and quantitation are additionally determined for degradation products to assist in establishment of the mass balance. 


\section{Stability indicating RP-HPLC/ HPLC approaches for different antiviral drugs}

In the present year, due to the appearing of new viruses, Hence of development and treatment of antiviral drugs also gain equal importance. Before the use of antiviral drugs should undergoes validation process to produce safe and efficient formulations. The various methods have been used for the quantitative determination of antiviral drugs such as UV, capillary electrophoresis, and Different chromatographic methods likes GC and HPLC, LC-MS, GC-MS. In this review, authors concentrate on stability, suggesting HPLC /RP-HPLC methods for the accurately and effective development and validation of selected antiviral drugs such as, Atazanavir sulfate[35-36], Abacavir[37-38], Acyclovir[39], Adefovir Dipivoxil[41], Boceprevir[42], Baloxavir marboxil[43],Cobicistat[44], Darunavir ethanolate[4546], Dolutegravir sodium[47-48], Didanosin[49], Efavirenz[50-52], Emtricitabine[53], Etravirine[54-55], Famciclovir[56-58], Foscarnet[59], Ganciclovir[60], Imiquimod[61], Lamivudine[62], Oseltamivir[63], Ribavirin[6465], Simeprevir[66-67], Tenofovir[68], Valganciclovir[69-72], Zanamivir[73] and Zidovudine[74].The selective stability indicating RP-HPLC/ HPLC approaches for different antiviral drugs is summaries in Table.2

\section{Conclusion}

Force degradation studies of drug substance give perceptive knowledge about the intrinsic stability of the molecule as well as possible degradants which formed during shelf life of drug and thus, aid within the successive development of its stable formulation. This review summarizes the current regulatory requirements for the practical performance of forced degradation and its application for the development of stability indicating method. There are numerous strategies have been implemented for the quantitative assessment of antiviral drugs. This study will provide detailed literature on stability- indicating HPLC/ RP-HPLC approaches for the development and validation of various antiviral drugs as well as gives a basic skill to the researchers who are working in the area of newer product development and quality control testing.

\section{Compliance with ethical standards}

\section{Acknowledgments}

Authors are thankful to Dr. Rajendra Gode College Pharmacy, Malkapur, for providing adequate facilities and unqualified support to carry out this review.

\section{Disclosure of conflict of interest}

The authors declare no conflict of interest, financial or otherwise.

\section{References}

[1] Zhang K, Pellett JD, Narang AS, Wang YJ, Zhang YT. Reactive impurities in large and small molecule pharmaceutical excipients-A review. TrAC Trends in Analytical Chemistry. 2018 Apr 1; 101: 34-42.

[2] Mallu UR, Nair AK, Bandaru S, Sankaraiah J. Hold time stability studies in pharmaceutical industry: Review. Pharm Reg Aff. 2012; 1(4): 1-8.

[3] Venkataraman S, Manasa M. Forced degradation studies: Regulatory guidance, characterization of drugs, and their degradation products-a review. Drug Invention Today. 2018 Feb 1; 10(2).

[4] Kaushik D, Bansal G. Characterization of degradation products of idarubicin through LC-UV, MSn and LC-MS-TOF studies. Journal of pharmaceutical and biomedical analysis. 2013 Nov 1; 85: 123-31.

[5] Rawat T, Pandey IP. Forced degradation studies for drug substances and drug products-scientific and regulatory considerations. Journal of pharmaceutical Sciences and research. 2015 May 1; 7(5): 238.

[6] Blessy MR, Patel RD, Prajapati PN, Agrawal YK. Development of forced degradation and stability indicating studies of drugs-A review. Journal of pharmaceutical analysis. 2014 Jun 1; 4(3): 159-65.

[7] Reynolds DW, Facchine KL, Mullaney JF, Alsante KM, Hatajik TD, Motto MG. Conducting forced degradation studies. Pharmaceutical technology. 2002 Feb; 48-56.

[8] Singh S, Junwal M, Modhe G, Tiwari H, Kurmi M, Parashar N, Sidduri P. Forced degradation studies to assess the stability of drugs and products. TrAC Trends in Analytical Chemistry. 2013 Sep 1; 49: 71-88. 
[9] Gendugov TA, Glushko AA, Ozerov AA, Shcherbakova LI. Study of the stability of the substance 3-[2-(4-phenyl-1piperazino)-2-oxoethyl] quinazoline-4 (3H)-one under stressful conditions. Pharmacy \& pharmacology. 2021 jan 31; 8(4): 242-54.

[10] Hotha KK, Reddy SP, Raju VK, Ravindranath LK. International research journal of pharmacy. Methods. 1: 10.

[11] Atkins M. Forced degradation studies comparison between ICH, EMA, FDA and WHO guidelines and ANVISA s resolution RDC. 53/2015.

[12] Chakole RD, Charde MS, Kumar J, Welankiwar AS. Review: Development of forced degradation studies of drugs. Int J Adv Pharm [Internet]. 2013 Jun 30; 2(3): 34-9.

[13] Iram F, Iram H, Iqbal AZHAR, Husain A. Forced degradation studies. J Anal Pharm Res. 2016; 3(6): 00073.

[14] Yasmeen A, Sofi G. A review of regulatory guidelines on stability studies. The Journal of Phytopharmacology. 2019; 8: 147-51.

[15] Klick S, Muijselaar PG, Waterval J, Eichinger T, Korn C, Gerding TK, Debets AJ, Sänger-Van De Griend C, Van Den Beld C, Somsen GW, De Jong GJ. Stress testing of drug substances and drug products. Pharm Technol. 2005 Feb; 29(2): 48-66.

[16] Guideline IH. Impurities in new drug substances Q3A (R2). InProceedings of the International Conference on Harmonization of Technical Requirements for Registration of Pharmaceuticals for Human Use, Geneva, Switzerland. 25 Oct 2006; 25.

[17] Maheswaran R. Scientific considerations of forced degradation studies in ANDA submissions. Journal of Validation Technology. 2012 Apr 1; 18(2): 92.

[18] Cartwright AC. 5 Quality Overall Summary. International Pharmaceutical Product Registration. 2016 Apr 19; 200: 78.

[19] Bhaskar R, Ola M, Agnihotri V, Chavan A, Girase H. Current Trend in Performance of Forced Degradation Studies for Drug Substance and Drug Product's. Journal of Drug Delivery and Therapeutics. 2020 Apr 15; 10(2-s): 14955 .

[20] EMEA C. Guideline on the limits of genotoxic impurities. Committee for Medicinal Products for Human Use (CPMP). 2006.

[21] Naveed S, Basheer S, Qamar F. Stability of a dosage form and forced degradation studies. J Bioequivalence Bioavailab. 2016; 8: 191-3.

[22] Tollefson AE, Hermiston TW, Lichtenstein DL, Colle CF, Tripp RA, Dimitrov T, Toth K, Wells CE, Doherty PC, Wold WS. Forced degradation of Fas inhibits apoptosis in adenovirus-infected cells. Nature. 1998 Apr; 392(6677): 72630 .

[23] Marın A, Barbas C. LC/MS for the degradation profiling of cough-cold products under forced conditions. Journal of pharmaceutical and biomedical analysis. 2004 Sep 3; 35(5): 1035-45.

[24] Alsante KM, Ando A, Brown R, Ensing J, Hatajik TD, Kong W, Tsuda Y. The role of degradant profiling in active pharmaceutical ingredients and drug products. Advanced drug delivery reviews. 2007 Jan 10; 59(1): $29-37$.

[25] Kats M. Forced degradation studies: regulatory considerations and implementation.2005.

[26] Carr GP, Wahlich JC. A practical approach to method validation in pharmaceutical analysis. Journal of pharmaceutical and biomedical analysis. 1990 Jan 1; 8(8-12): 613-8.

[27] Roge AB, Tarte PS, Kumare MM, Shendarkar GR, Vadvalkar SM. Forced degradation study: an important tool in drug development. Asian Journal of Pharmaceutical Research. 2013 Oct; 3(4): 198-201.

[28] Adhao VS, Chaudhari SR, Ambhore JP, Sangolkar S, Thenge RR, Cheke RS, Patil AS. Reverse phase-liquid chromatography assisted protocol for simultaneous determination of lamivudine and tenofovir disoproxil fumarate in combined medication used to control HIV infection: an investigative approach. Future Journal of Pharmaceutical Sciences. 2021 Dec;7(1):1-1.

[29] Patel NN, Kothari CS. Critical review: Significance of Force degradation study with respect to current Pharmaceutical Scenario. Asian Journal of Research in Chemistry. 2013 Mar 1; 6(3): XII.

[30] Bakshi M, Singh S. Development of validated stability-indicating assay methods-critical review. Journal of pharmaceutical and biomedical analysis. 2002 Jun 15; 28(6): 1011-40. 
[31] Dolan JW. Stability-indicating assays. LC GC North America. 2002; 20(4): 346-9.

[32] Qiu F, Norwood DL. Identification of pharmaceutical impurities. Journal of liquid chromatography \& related technologies. 2007 Feb 1; 30 (5-7): 877-935.

[33] Annapurna MM, Mohapatro C, Narendra A. Stability-indicating liquid chromatographic method for the determination of Letrozole in pharmaceutical formulations. Journal of pharmaceutical analysis. 2012 Aug 1; 2(4): 298-305.

[34] Guideline IHT. Text on validation of analytical procedures. International Conference on Harmonization, Geneva. October 1994; 1-5.

[35] Dey S, Patro SS, Babu NS, Murthy PN, Panda SK. Development and validation of a stability-indicating RP-HPLC method for estimation of atazanavir sulfate in bulk. Journal of pharmaceutical analysis. 2017 Apr 1; 7(2): 13440 .

[36] Bhirud CH, Hiremath SN. Stability indicating RP-HPLC method for the determination of Atazanavir sulphate in bulk and dosage form. Drug Invention Today. 2013 Jun 1; 5(2): 81-6.

[37] Kumar P, Dwivedi SC, Kushnoor A. A validated stability indicating RP-HPLC method for the determination of tenofovir in bulk and tablet dosage forms. Eurasian Journal of Analytical Chemistry. 2012 Jan 15; 7(2): 104-14.

[38] Kiran BV, Rao BS, Dubey SS. Validation of Abacavir Sulfate In Pharmaceutical Dosage By Reverse Phase HPLC With Internal Standard Method. International Journal of pharmaceutical sciences and research. 2012 Aug 1; 3(8): 2590 .

[39] Chaudhari V, Ubale M. A validated stability-indicating HPLC assay method for acyclovir in bulk drug. Int J Anal Pharm Biomed Sci. 2012; 1: 5-12.

[40] Gunasekar M, Development and Validation of a Stability-Indicating RP-HPLC Method for the Estimation of Aciclovir, in Bulk and Ointment Dosage Form. International Journal of Pharmaceutical and Phytopharmacological Research. 2019 Feb; 9(1): 1-18.

[41] Darsazan B, Shafaati A, Mortazavi SA, Zarghi A. A simple and specific stability-indicating RP-HPLC method for routine assay of adefovir dipivoxil in bulk and tablet dosage form. Iranian journal of pharmaceutical research: IJPR. 2017; 16(1): 132.

[42] Damle MC, Salunke SR. Development and validation of stability-indicating HPLC method for determination of boceprevir. International Research Journal of Pharmacy. 2016; 7(8): 47-53.

[43] Raveendranath TV, Saravanakumar R, Anjana C. Development and Validation of Stability Indicating RP-UPLC Method for the Quantification of Baloxavir Marboxil in Tablet Formulation. International Journal of Pharmacy and Pharmaceutical Sciences. 2020 Nov 1; 94-9.

[44] Kalyani K, Anuradha V. A stability-indicating high performance liquid chromatographic method for the determination of cobicistat. International Journal of Pharmaceutics and Drug Analysis. 2015 Apr 24; 117-25.

[45] Reddy BR, Jyothi G, Reddy BS, Raman NV, Reddy KS, Rambabu C. Stability-Indicating HPLC method for the determination of darunavir ethanolate. Journal of chromatographic science. 2013 May 1; 51(5): 471-6.

[46] Corrêa JC, Serra CH, Salgado HR. Stability study of darunavir ethanolate tablets applying a new stability-indicating HPLC method. Chromatography Research International. 15 May 2013.

[47] Bhavar GB, Pekamwar SS, Aher KB, Thorat RS, Chaudhari SR. High-Performance Liquid Chromatographic and High-Performance Thin-Layer Chromatographic Method for Quantitative Estimation of Dolutegravir Sodium in Bulk Drug and Pharmaceutical Dosage Form. Scientia pharmaceutica. 2016 Jun; 84(2): 305-20.

[48] Venkatnarayana M, Siva Jyothi N. Development of Validation and Stability Indicating Method of Anti-HIV Dolutegravir Drug and its Related Impurities by Using RP-HPLC. J Chromatogr Sep Tech. 2020; $11: 426$.

[49] Gholve S, Gangapure S, Birajdar M, Mujewar I, Bhusnure OG. RP-HPLC Method Development and Validation for Determination of Didanosine in Pharmaceutical Dosage Forms. Journal of Drug Delivery and Therapeutics. 2019 Aug 15; 9(4-s): 343-7.

[50] Nathi R, Rao SS, Sahoo S, Sunkara N, Mohan VR. Stability Indicating RP-HPLC Method Development and Validation of Efavirenz in Bulk and Pharmaceutical Dosage Form. 
[51] Hamrapurkar PD, Patil PS, Phale MD, Shah N, Pawar SB. Optimization and validation of RP-HPLC stabilityindicating method for determination of efavirenz and its degradation products. International Journal of Applied Science and Engineering. 2010 Dec 1; 8(2): 155-65.

[52] Ambhore JP, Chaudhari SR, Cheke RS, Kharkar PS. A Concise Analytical Profile of Efavirenz: Analytical Methodologies. Critical Reviews in Analytical Chemistry. 27 Feb 2021; 1-0.

[53] Seshachalam U, Haribabu B, Chandrasekhar KB. Development and validation of a stability-indicating liquid chromatographic method for determination of emtricitabine and related impurities in drug substance. Journal of separation science. 2007 May; 30(7): 999-1004.

[54] Murali D, Rambabu C. Development and validation of stability indicating RP-HPLC method for the quantification of etravirine in tablet dosage form.

[55] Phase Df. Analytical Method Development and Validation Of Etravirine In Its Bulk Dosage Form By Using Reverse Phase High Performance Liquid Chromatography Method As Per International Conference On Harmonisation Guidelines. Asian J Pharm Clin Res. 2015; 8(2): 147-50.

[56] Patil DD, Fursule RA. A Stability Indicating LC Method for Famciclovir. Research Journal of Pharmacy and Technology. 2010 Sep 28; 3(3): 762-5.

[57] Raju CB, Panda G, Rao GN. Stability indicating LC assay method for the determination of famciclovir in bulk drug and pharmaceutical dosage forms. Chromatographia. 2008 Nov; 68(9): 837-41.

[58] Kumar MN, Reddy VK, Sharma HK, Mastanaiah T. Simple and reliable stability indicating RP-HPLC method for the determination of assay of famciclovir in famciclovir drug substance. Der Chem Sin. 2012; 3: 450-7.

[59] Goud VM, Rao AS. Stability indicating RP-HPLC method development and validation of foscarnet in bulk and pharmaceutical dosage form. Der Pharm Lett. 2015; 7: 1-6.

[60] Madhusudhan Patlola VR, Singh S. Determination of Assay and Validation of Stability Indicating RP-HPLC Method for Ganciclovir in nciclovir Drug Substance. International Journal of Scientific \& Technology Research.2020; 9(2):4191-4195.

[61] Hussain, Sayyed \& Shaikh, Tabrez \& Farooqui, Mazahar. Development and Validation of Liquid Chromatography Method for the Determination and Quantification of Impurities in Imiquimod. British Journal of Pharmaceutical Research. 2016; 13: 1-9.

[62] Saibaba Sv, Shanmuga P. Development and Validation of a Stability indicating HPLC method for the Determination of Lamivudine in Bulk and Pharmaceutical dosage form. International Journal of Pharma Sciences and Research. 2016; 7: 471-477.

[63] Narasimhan B, Abida K, Srinivas K. Stability indicating RP-HPLC method development and validation for oseltamivir API. Chemical and Pharmaceutical Bulletin. 2008 Apr 1; 56(4): 413-7.

[64] Haggag RS, Belal SF, Hewala II, El Rouby OA. Stability-indicating HPLC-DAD determination of ribavirin in capsules and plasma. Journal of chromatographic science. 2014 Jul 1; 52(6): 493-500.

[65] Belal F, Sharaf El-Din MK, Eid MI, El-Gamal RM. Validated stability-indicating liquid chromatographic method for the determination of ribavirin in the presence of its degradation products: application to degradation kinetics. Journal of chromatographic science. 2015 Apr 1; 53(4): 603-11.

[66] Attia KA, El-Abasawi NM, El-Olemy A, Serag A. Stability-indicating HPLC-DAD Method for the Determination of Simeprevir. Analytical Chemistry Letters. 2017 Jan 2; 7(1): 43-51.

[67] Vanitha C, Satyanarayana Sv, \& Bhaskar Reddy K. Quality By Design Approach To Stability-Indicating ReversePhase High-Performance Liquid Chromatography Method Development, Optimization, And Validation For The Estimation Of Simeprevir In Bulk Drug. Asian Journal of Pharmaceutical and Clinical Research. 93-100.

[68] Suvarna VM, Sangave PC. Development and validation of stability indicating rp-hplc method for tenofovir solid lipid nanoparticles. International Journal of Pharmaceutical Sciences and Research. 2017 Feb 1; 8(2): 658.

[69] Mondal S, Reddy GS, Mondal P, Prathyusha VS, Aishwarya PN, Rahaman ST. A new stability indicating ultraperformance liquid chromatography-PDA method for the estimation of valganciclovir in bulk and tablet dosage form. Pharm Methods. 2018; 9: 94-8. 
[70] Sawant S, Barge V. A validated stability indicating RP-HPLC method for valganciclovir, identification and characterization of forced degradation products of valganciclovir using LC-MS/MS. Acta Chromatographica. 2014 Mar 2014; 26(1): 29-42.

[71] Veni K, Gowramma B, Madhuri L, Gouthami B, Nag S, Meyyanathan SN. Development and validation of a stability indicating RP-HPLC method for the determination of valganciclovir hydrochloride (RS). Res Rev J Pharm Anal. 2014; 3: 19-26.

[72] Ramesh G, Rao MS. Development and validation of stability indicating RP-HPLC method for quantitative determination of valganciclovir in pure and pharmaceutical formulations. Int JPharm Pharm Res. 2015; 3: 1-14.

[73] Bhirud CH, Nandal DH. Stability indicating RP-HPLC and HPTLC methods for the determination of zanamivir in bulk and dosage form. Int J Pharm Pharm Sci. 2016; 8(7): 249-56.

[74] Mandloi D, Tripathi P, Mohanraj P, Chauhan NS, Patel JR. Development and validation of a stability-indicating hplc method for analysis of zidovudine (zdv) in bulk drug and in vitro release studies of tablets. Journal of liquid chromatography \& related technologies. 2011 Apr 12; 34(8): 601. 